Technological University Dublin ARROW@TU Dublin

\title{
RF Breast Cancer Detection Employing a Non-characterized Vivaldi Antenna and a MUSIC-Inspired Algorithm
}

\author{
Giuseppe Ruvio \\ Technological University Dublin, Giuseppe.Ruvio@tudublin.ie \\ Raffaele Solimene \\ Seconda Università di Napoli \\ Antonietta D'Alterio \\ Seconda Università di Napoli
}

See next page for additional authors

Follow this and additional works at: https://arrow.tudublin.ie/ahfrcart

Part of the Biomedical Engineering and Bioengineering Commons

\section{Recommended Citation}

G. Ruvio, R. Solimene, A. D'Alterio, M. J. Ammann, and R. Pierri, "RF Breast Cancer Detection Employing a Non-characterized Vivaldi Antenna and a MUSIC-like Algorithm", International Journal of RF and Microwave Computed-Aided Engineering, vol. 23, issue 5, pp. 598-609, 09/2013.doi:10.1002/mmce.20694

This Article is brought to you for free and open access by the Antenna \& High Frequency Research Centre at ARROW@TU Dublin. It has been accepted for inclusion in Articles by an authorized administrator of ARROW@TU Dublin. For more information, please contact arrow.admin@tudublin.ie, aisling.coyne@tudublin.ie, gerard.connolly@tudublin.ie.

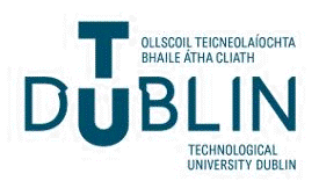


Authors

Giuseppe Ruvio, Raffaele Solimene, Antonietta D'Alterio, Max Ammann, and Rocco Pierri

This article is available at ARROW@TU Dublin: https://arrow.tudublin.ie/ahfrcart/54 


\title{
RF Breast Cancer Detection Employing a Non-characterized Vivaldi Antenna and a MUSIC-Inspired Algorithm
}

\author{
Giuseppe Ruvio, Raffaele Solimene, Antonietta D’Alterio, Max J. Ammann, and Rocco Pierri
}

\begin{abstract}
A novel microwave breast cancer detection system consisting of an Evolutionary Global Optimized Vivaldi antenna and an algorithm inspired by MUltiple SIgnal Classification (MUSIC) is presented. Its performance is assessed by using a simplified numerical breast phantom for a number of critical conditions including the presence of fibroglandular tissues.
\end{abstract}

\section{Index Terms}

Electromagnetic inverse scattering, linear inverse problems

\section{INTRODUCTION}

Breast cancer is the most common cancer in women and X-ray mammography is the most widely used diagnostic technology in mass screening. However, its detection capabilities have been shown to be limited due to poor benign/malignant tissue contrast. Indeed, between $4 \%$ and $34 \%$ of all breast cancers are missed and nearly $70 \%$ of all breast lesions turn out to be benign [1]. In addition, as X-ray mammography requires breast compression, chest wall adjacent regions are left unscreened. Furthermore, the exposure to low levels of ionizing radiation may reduce patient compliance with screening recommendations and limit frequent check-ups.

These concerns motivated the search for techniques that image other physical tissue properties or metabolic changes. Among them, active microwave based diagnostic techniques have triggered great interest. At UHF frequencies, normal and malignant breast tissues exhibit a sharper dielectric contrast which can be greater than $2: 1$, i.e., notably superior to the radiographic density exploited by X-ray mammography [2]. At the same time, the need for breast compression and ionizing screening are eliminated.

Over the last two decades, significant research activities were focused on the early detection of breast cancer by microwave imaging which led to several techniques for detecting, localizing and characterizing tumors in breast tissues. Such methods can be broadly grouped into two categories: one which aims at reconstructing the dielectric and conductivity maps of the breast and another which detects and localizes in-homogeneities in the breast. In both cases, an electromagnetic inverse scattering problem exists [3].

In the first case the intrinsic non-linearity of inverse scattering problems is taken into account. Accordingly, retrieving the dielectric and conductivity profiles entails running optimization schemes which are computationally intensive [4]. Those imaging solutions also suffer from reliability problems due to the occurrence of false solutions. This is a common consequence of deterministic minimization algorithms [5].

For the second class, simplified scattering models are employed, so that the detection problem requires linear procedures. Delay and sum procedure (and their different variants [6]), time-reversal algorithm [7] and the distributional approach [8] are a few of focusing algorithms based on linear inversion. Of course, as simplified models are employed, only "qualitative" information can be gathered and, hence, a further classification step is generally needed [9]. However, problems of reliability and computational burden are avoided.

In 2007 Lazebnik [10], [11] conducted a large scale study to experimentally determine the microwave dielectric properties of a variety of normal, malignant and benign breast tissues obtained from cancer surgeries. Those results stimulated improvement of beam-forming algorithms to take into account the reduced benign/malignant breast tissue dielectric contrast. Dielectrically heterogeneous MRI-derived breast models and ideal radiating elements were used to assess tumor localization for different radar configurations and contrast enhancement techniques. However, if ideal sources are very useful to promptly evaluate imaging algorithms, the design of the front-end of an RF breast cancer imaging system has fundamental importance for the overall performance. In particular, antennas must fulfil specific requirements including operating band, field penetration inside the breast, phase-center stability, compactness and cost.

Ultra-Wideband (UWB) antennas emerge as suitable candidates for this application due to their properties such as large operating band, phase-linearity and compactness. Therefore, different antenna geometries have been proposed in literature.

G. Ruvio and M. J. Ammann are with the Antenna and High Frequency Research Centre, Dublin Institute of Technology, Kevin Street, Dublin 8, Ireland.

R. Solimene, A. D'Alterio and R. Pierri are with Seconda Università di Napoli, Dipartimento di Ingegneria dell'Informazione, via Roma 29, I-81031 Aversa, Italy. 


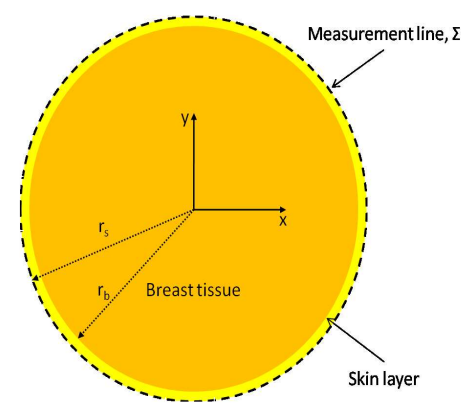

Fig. 1. Configuration layout.

Planar monopole antennas are able to combine a miniaturized low-profile design with very broad impedance matched bandwidth and good stability when placed close to the breast [12], [13]. However, their omnidirectional behavior can lead to interference coming from unwanted directions. The same drawback might affect a dipole antenna placed conformally around the breast phantom as investigated in [14]. Horn antennas have been also proposed for their large operating bandwidth and directive pattern [15]. But their bulky structure is suitable just for multi-monostatic configurations with only one TX/RX antenna that moves across a certain number of scanning positions. In [16], [17] a wide-slot antenna is introduced as a radiator for a 31-element conformal array. Its size is affordable in the frequency range $4-8 \mathrm{GHz}$, where the limited field penetration is compensated for the large number of array elements. Vivaldi antennas combine a large impedance matching bandwidth together with a directive pattern, so that they can divert more power towards the target and reduce backscattering coming from obstacles outside the volume under investigation. In [18] Vivaldi antennas are miniaturized by corrugating the external flanges to operate as breast cancer sensors in the frequency range 3.1 - 6.85 GHz. Efficient Global Optimization (EGO) techniques can also be employed in order to improve their UWB features [19], [20]. EGO optimisation was used in [13], where a planar antipodal Vivaldi antenna is evaluated in terms of operating bandwidth, fidelity factor and field penetration into a planar breast phantom.

In this paper a novel breast cancer detection system consisting of a planar antipodal Vivaldi antenna and a detection algorithm inspired based by MUltiple SIgnal Classification (MUSIC) [21] is presented. As an a priori characterization of the antenna is not required, a complex optimized geometry can be chosen without the necessity of further algorithm tailoring. The performance of such antenna + algorithm system can be compared to what was obtained by using an ideal dipole source as presented in [22]. In particular, the antenna phase response is not required to be known a priori, neither is this calibrated before achieving tumor detection. Indeed, such characterization is not required at all in any detection stage of the proposed procedure. In order to focus on this aspect, a simplified breast model is considered, although in combination with a non-ideal antenna as commonly done in the literature [14], [23], [24]. When the antenna is located close to an unknown breast structure, its behavior changes and its a priori characterization becomes extremely difficult. This issue dramatically reduce the performance of RF tomographic techniques that require coherent data where at least the antenna phase frequency response must be known for obtaining properly focused reconstructions. A MUSIC-inspired detection algorithm is here proposed to address this problem of great relevance in practical situations and its performance is assessed across a large number of scenarios. In particular, the analysis encompasses the cases where:

a single tumor is located at different position within the breast tissue;

the co-presence of three tumors which are positioned at different distances from the skin layer;

fibroglandular structures are also considered for critical benign/malignant tissue contrast.

As the primary task of this investigation is to detect and localize tumors inside the breast tissue, a linear model in conjunction to a MUSIC-like algorithm is adopted to develop detection algorithm. This avoids the disadvantages associated with non-linear optimization. In particular, a multi-monostatic/multifrequency configuration is employed.

The paper is organized as follows. Sections II, III and IV, are respectively devoted to the introduction of the adopted phantom, the antenna design and the detection algorithm description. Numerical analysis is discussed in Section V.

\section{The Phantom}

The cancer detection problem is addressed by considering the simplified breast phantom displayed in Fig. 1. It consists of a coaxial cylindrical structure with a diameter $2 r_{s}=100 \mathrm{~mm}$ which simulates the skin and breast tissue layers. The tumor is a smaller cylinder of 5 -mm diameter that is inserted inside the breast tissue.

The phantom is immersed in a coupling medium with a relative dielectric permittivity $\epsilon_{m}=10$. Moreover, it is 400 mm long (i.e., more than 4 times the wavelength $\lambda$ in the coupling medium at the lower edge frequency of $1 \mathrm{GHz}$ ) in order to prevent the impact of unwanted diffractive effects on the fields collected at the central cross-section of the phantom. 


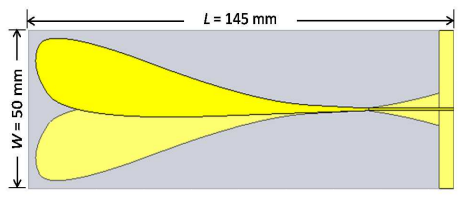

Fig. 2. Geometry of the antipodal Vivaldi antenna.

The electromagnetic properties of skin, benign and malignant mammary tissues have been set according to [25]. In particular, the skin thickness $r_{b}-r_{s}$ is $2 \mathrm{~mm}$, which is much lower than wavelength in medium. Accordingly, this is modelled by using just a conductivity $\sigma_{s}$ of $4 \mathrm{~S} / \mathrm{m}$ and a relative dielectric permittivity $\epsilon_{s}$ of 36. The breast tissue is represented by using a first order Debye model $\epsilon_{b}=\epsilon_{\infty b}+\left(\epsilon_{\infty b}-\epsilon_{s b}\right) /\left(1+j \omega \tau_{b}\right)+\sigma_{b} /(j \omega)$ with $\epsilon_{s b}=10, \epsilon_{\infty b}=7, \sigma_{b}=0.15 \mathrm{~S} / \mathrm{m}$ and relaxation time $\tau_{b}=7.0 \mathrm{ps}$. In the same fashion, the electromagnetic properties of the tumor tissue are given in terms of a Debye model with $\epsilon_{s c}=54, \epsilon_{\infty c}=4, \sigma_{c}=0.7 \mathrm{~S} / \mathrm{m}$ and $\tau_{c}=7.0 \mathrm{ps}$. Finally, for the cases where fibroglandular tissues are present, they have been modelled as in [26] to be between $10 \%$ and $50 \%$ electromagnetically denser than breast tissue. This corresponds to the electric properties spanning in the ranges $\epsilon_{s f g}=11-15, \epsilon_{\infty f g 10 \%}=7.7-10.5, \sigma_{f g 10 \%}=0.165-0.225 \mathrm{~S} / \mathrm{m}$, whereas the relaxation time $\tau_{f g}$ is kept constant at $7.0 \mathrm{ps}$.

\section{ANTENNA DESIGN}

Since antenna calibrations or phase response estimations are not required by the detecting algorithm, efficient and complex antenna geometries can be designed for improved coupling with mammary tissues. Large freedom is this way enabled to the antenna designer. In addition, a more relaxed broadband antenna behavior compared to other UWB approaches is affordable due to the fact that the system operates on a discrete set of frequencies rather than on continuous spectrum.

An antipodal planar Vivaldi antenna was chosen for its compactness, directive behavior and broad impedance matching properties. In Fig. 2 the geometry of the Vivaldi antenna along with its characteristic parameters $L$ and $W$, which define the overall geometry and strongly affect the antenna's resonances, are shown.

The antenna is designed to work immersed in a coupling medium that presents dielectric properties which are similar to the breast tissue (see previous section) in the frequency band of interest. While this solution enables more energy to be coupled between the antenna and the phantom, a strong degree of miniaturization can be also achieved. Moreover, as we are operating in the frequency range greater than $1 \mathrm{GHz}$, the antenna side $W$ placed against the skin layer, can be kept small. This choice is very convenient when a number of sensors -(up to 18 in this paper)- have to be positioned around the breast phantom.

The design of the printed antipodal Vivaldi antenna has been aided by the computationally efficient multiobjective evolutionary algorithm ParEGO [27]. As a result of a 150 evaluation loop, the algorithm controls the generator points of the spline-based arms for an optimized return loss not greater than $8 \mathrm{~dB}$ between $1-3 \mathrm{GHz}(100 \%$ bandwidth) with the antenna fully immersed into the coupling medium. A solution of water and paraffin oil can be used for this purpose as suggested in [14].

The antenna has been realized with a $1.58 \mathrm{~mm}$ thick Taconic CER10 substrate, which presents a stable dielectric constant equal to 10 across the operating frequency range.

The behavior of the antenna positioned against a similar phantom was studied in [13]. It was observed that the phantom has a loading effect on the antenna that corresponds to an overall improved matching over the frequencies of interest.

\section{DETECTION ALGORITHM}

The scattered field data are collected under a multi-monostatic configuration (i.e. TX and RX are co-located) over a circle $\Sigma$ of radius $r_{s}=50 \mathrm{~mm}$ in correspondence to $N$ measurement positions $\left(\underline{r}_{o 1}, \underline{r}_{o 2}, \cdots, \underline{r}_{o N}\right)$.

As mentioned above, we are mainly interested in the detection and the localization of small (with respect to the wavelength) tumors. Therefore, the field scattered by each single tumor can be represented in terms of low frequency model, where only the first order multipole can be considered as relevant [28]. Moreover, as only qualitative information is of concern (i.e. the degree of contrast of the scatterer is not required for the detection procedure), while developing the imaging algorithm, we neglect mutual scattering, so that a linear model can be conveniently adopted for the scattering phenomenon [8].

Accordingly, given $M$ the number of scatterers located at $\left(\underline{r}_{1}, \underline{r}_{2}, \cdots, \underline{r}_{M}\right)$, (with $N>M$ ), the scattered field vector can be written as 


$$
\underline{E}_{S}(f)=\underline{\underline{A}}(f) \underline{b}(f)
$$

where $f$ is the working frequency, $\underline{b}(f)$ is the $[M, 1]$ vector of the in-homogeneities scattering coefficients and $\underline{A}(f)$ is the $[N, M]$ propagator which maps the scatterers to the scattered field. In particular, its $n$-th column has the form

$$
\underline{A}^{n}\left(\underline{r}_{n} ; f\right)=\left[G^{2}\left(\underline{r}_{o 1}, \underline{r}_{n} ; f\right), G^{2}\left(\underline{r}_{o 2}, \underline{r}_{n} ; f\right), \cdots, G^{2}\left(\underline{r}_{o N}, \underline{r}_{n} ; f\right)\right]^{T}
$$

$G(\cdot)$ being the Green's function corresponding to a homogeneous medium having the same electromagnetic parameters as the breast tissue, hence skin is not accounted for.

From eq. (1) it can be seen that, while the scattering coefficient vector accounts for the objects' contrasts, information concerning their locations is embodied within the columns of $\underline{\underline{A}}(f)$. Therefore, as we are interested in retrieving such positions, disregarding contrast characterizations, a subspace projection method like MUSIC [21] can be adopted.

To this end, the correlation matrix is built up as follows

$$
\underline{\underline{R}}(f)=\underline{E}_{S}(f) \underline{E}_{S}^{H}(f)=\underline{\underline{A}}(f) \underline{\underline{B}}(f) \underline{\underline{A}}^{H}(f)
$$

where $\underline{b}^{H}(f)$ and $\underline{\underline{A}}^{H}(f)$ are the Hermitian vector and matrix of $\underline{b}(f)$ and $\underline{\underline{A}}(f)$, respectively, and $\underline{\underline{B}}(f)=\underline{b}(f) \underline{b}^{H}(f)$.

According to [21], scatterers' locations can be identified by finding the steering vectors which are orthogonal to the socalled noise subspace. This requires computing the eigen-spectrum of $\underline{\underline{R}}(f)$ and the steering vectors consisting of columns $\underline{A}_{k}(f)=\underline{A}^{k}\left(\underline{r}_{k} ; f\right) /\left\|\underline{A}^{k}\left(\underline{r}_{k} ; f\right)\right\|$ evaluated in correspondence to the trial position $\underline{r}_{k}$.

However, here, as the scattering coefficient vector is deterministic, $\underline{B}(f)$ and hence $\underline{\underline{R}}(f)$ are rank-deficient with rank one. This suggests to adopt the only significant singular vector $\underline{u}_{1}(f)$ of $\underline{\underline{R}}(f)$ to implement the detection algorithm. Accordingly, the following scheme to identify the scatterers' locations is used

$$
P\left(\underline{r}_{k} ; f\right)=1 /\left|\log \left(\left|<\underline{A}_{k}(f), \underline{u}_{1}(f)>\right|\right)\right|
$$

where $\underline{A}_{k}$ is evaluated at the trial position $\underline{r}_{k}$ according to the definition given above and $\langle\cdot, \cdot\rangle$ denotes the Hermitian scalar product. Accordingly, detection is achieved for non-zero values of the indicator function $P(\cdot)$.

As $\underline{\underline{R}}(f)$ is rank deficient some artifacts can appear in the reconstructed image. To avoid this inconvenience, some methods to restore the rank of the correlation matrix in conjunction with multifrequency illumination [29] or a multistatic configuration [30] can be employed. Both methods require coherence between the different data acquisitions. However, while the antenna response is known in free-space, when in close proximity to breast tissue, the response changes. That is why the singlefrequency approach is considered in (4). To counteract artifacts a multiple frequencies version of MUSIC as in can be adopted as in [31]. Here, we propose to modify the detection scheme by accommodating multifrequency data as

$$
P_{m f}\left(\underline{r}_{k}\right)=1 / \mid \log \left(\prod_{n=1}^{N_{f}}\left|<\underline{A}_{k}\left(f_{n}\right), \underline{u}_{1}\left(f_{n}\right)>\right|\right)
$$

where $N_{f}$ is the number of adopted frequencies and $f_{n}$ is the $n$-th frequency.

It is expected that beating projections at different frequencies helps in reducing the occurrence of spurious artifacts due to reduced rank of $\underline{\underline{R}}$.

Although, a simplified phantom was adopted, it includes sources of in-homogeneity (i.e. skin and fibroglandular structures) that are not considered in the Green's function definition. Regarding the homogeneity assumption and matrix $\underline{A}_{k}$ definition, we remark that only the correlation matrix $\underline{\underline{R}}$ and the adopted steering vector are relevant in the detection stage. While the steering vector is actually formed according to the homogeneity assumption, the correlation matrix is not. Indeed, $\underline{\underline{R}}$ is constructed starting from measurements which take into account the actual breast configuration. The scenarios under investigation always refer to an in-homogeneous breast due to the presence of the skin layer and, in most cases, fibrograndular structures. Moreover, these in-homogeneities are never considered in the steering vector formation.

As a concluding remark it is remarked that what is actually collected to perform reconstruction is the scattering parameter $S_{11}\left(\underline{r}_{o n}, f\right)$. Therefore, in order to obtain the scattered field, data must be calibrated by compensating for the the antenna frequency response in transmission $H_{t}(f)$ and reception $H_{r}(f)$. However, due to the adopted multimonostatic configuration and the incoherent strategy adopted in eq. (5) $H_{t}(f)$ and $H_{r}(f)$ do not play any role while achieving detection. This is a very important features of the presented detection scheme as antenna frequency response become itself unknown when sensors are located in close proximity of an unknown breast. 
a
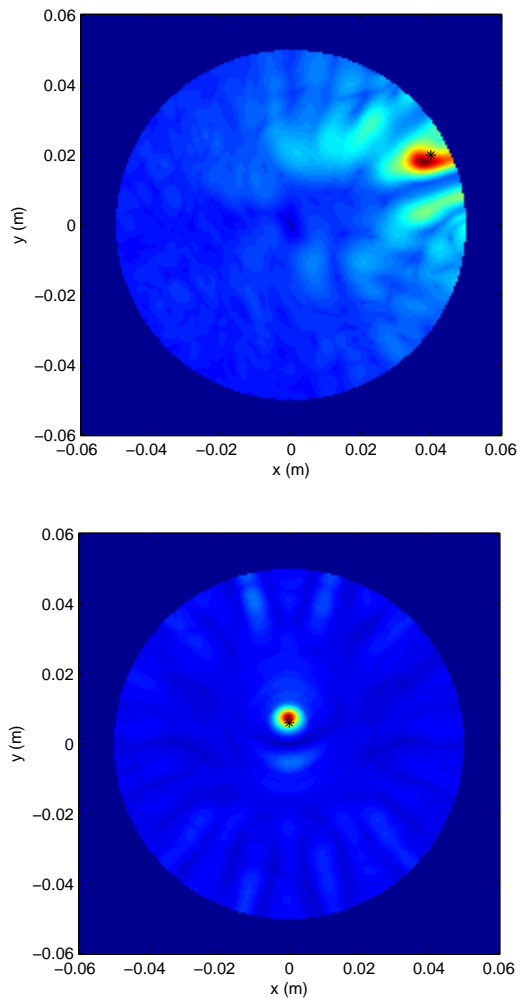

c b

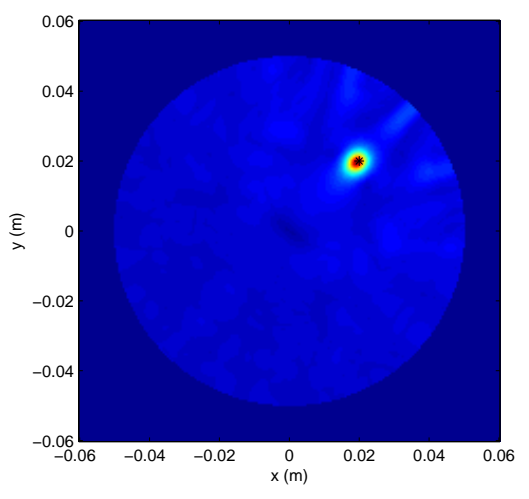

Fig. 3. Detection of a tumor for different distances from the skin. Black marker denotes actual tumor's center.

\section{NUMERICAL INVESTIGATION}

The detection system whose functional parts have been described in the previous sections is now tested in different scenarios. Each case considered corresponds to a stressing condition for the detection algorithm and aims to evaluate the robustness of the full system. In particular, synthetic data have been generated by CST Microwave Studio [32] by collecting the scattered field over 12 or 18 different positions taken uniformly around the breast phantom at multiples of $30^{\circ}$ or $20^{\circ}$, respectively, whereas 10 beating frequencies within the band $1-3 \mathrm{GHz}$ are adopted. In order to evaluate the performance achievable by the detection system different metrics are employed:

Full-Width-Half-Maximum (FWHM);

Signal-to-Clutter Ratio (SCR);

Signal-to-Mean Ratio (SMR);

Spatial displacement (SD).

The FWHM expresses the degree of resolution and is given by twice the distance between the peak value in the reconstruction and the points where the energy drops to half the maximum value, averaged over the different directions. The SCR and SMR compare the maximum tumor response in the reconstructed image with the maximum and the average clutter response in the same image, respectively. In particular, clutter is evaluated outside the tumor region and is estimated as twice the FWHM. The $\mathrm{SD}$ measures the error in tumor localization and accounts for the difference between the position of the tumor as peak value in the reconstruction and as actual center position in the scanned phantom [24].

In order to obtain the scattered field, clutter is removed by subtracting the average trace from those collected by each sensor. Finally, we outline that, all cases presented below have been obtained by corrupting the scattered field with an additive white complex Gaussian noise with signal-to-noise ratio $S N R=10 \mathrm{~dB}$.

\section{A. Presence of one tumor}

The system detection capability has been initially tested in the presence of one $5 \mathrm{~mm}$-diameter tumor and the Vivaldi antenna scanning the phantom across 12 positions spaced $30^{\circ}$ from each other.

In order to obtain a general understanding of the achievable performance the tumor has been positioned in three different locations.

More specifically, a single malignant in-homogeneity has been placed at the coordinates (40, 20) mm (i.e., just below the skin tissue), at $(20,20) \mathrm{mm}$ and $(0,6) \mathrm{mm}$, respectively. All the positions are referred to the center of the coaxial structure and are addressed as position $a, b$ and $c$ in Fig. 3 . 
TABLE I

METRICS FOR THE CASE OF A SINGLE TUMOR.

\begin{tabular}{|c|c|c|c|c|}
\hline Metrics & FWHM $(\mathrm{mm})$ & SCR $(\mathrm{dB})$ & SMR $(\mathrm{dB})$ & SD $(\mathrm{mm})$ \\
\hline Position a & 7.2 & 5.9 & 14.4 & 2.8 \\
\hline Position b & 5 & 14.7 & 22.4 & 0.99 \\
\hline Position c & 4 & 12.7 & 19.3 & 1.2 \\
\hline
\end{tabular}

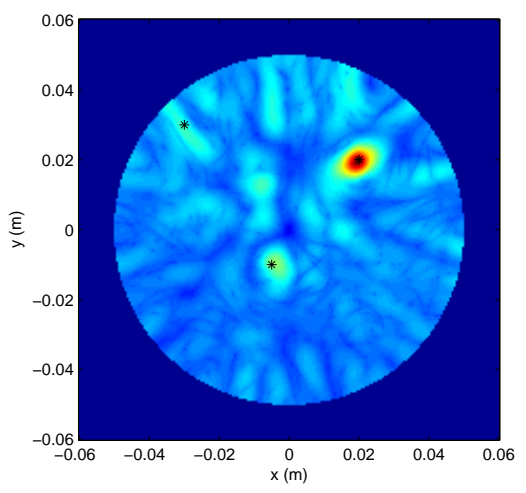

a

Fig. 4. Detection of three tumors simultaneously present within the breast and located at different distances from the skin. Black markers denote actual tumor positions. a: reconstruction obtained by 12 sensors; b: reconstruction obtained by 18 sensors.

Position $a$ takes into account the least favorable scenario for detection as very few scans contribute in reconstructing the target. In fact, for most of the scans, the distance between the antenna and the tumor is very large and the related scattered signals are strongly attenuated. As illustrated in Fig. 3a, the detection is correctly performed, although a certain loss of resolution can be also observed. Compared to the previous case, position $b$ represents a more balanced scenario as the antenna gets close to the tumor in a larger number of scans. From Fig. 3b it can be observed that the maximum of the reconstruction occurs where the tumor center lies as positioned in the phantom (black marker). Finally, position $c$ represents a particular case for the tumor being located very close to the center of the scanning rotation. This corresponds to a stress situation for the system as the algorithm works close to an ambiguity condition (i.e. equal scattered signal detected at each scan). However, also for this case, the tumor is correctly detected with high accuracy and low artifacts level.

Table I gives a quantitative measure of the achievable performance for the three positions considered above. It can be observed that the tumor size in the third case is underestimated. This can occur as the detection algorithm estimates the tumor centers disregarding their size. This explains the FWHM results obtained as this parameter gives a measure on how accurate is such center estimation rather then the tumor size.

\section{B. Presence of three tumors}

In this section the detection system has been tested against a scattering scenario where three tumors are simultaneously present. The aim is to address a situation consisting of a combination of the challenging scenarios from previous sections.

The three tumors are located in $(20,20) \mathrm{mm},(-25,25) \mathrm{mm}$ and $(-5,-10) \mathrm{mm}$, respectively.

First, we consider the same configuration with 12 scanning positions as above. The corresponding reconstruction is reported in Fig. 4a. It can be seen that all not all targets are discernable from clutter artifacts detected and well localized. However,In particular the tumor located under the skin and the one close to the breast center are more weakly reconstructed. This could be expected as it is consistent with previous results. This situation can be largely improved by adding more sensor positions. -, for example, by rotating the whole system as in [?]-. The reconstruction corresponding to the number of sensor positions increased up to 18 is shown in the same figure on right panel. It can be now observed that all tumors are more discernible against clutter artifacts.

\section{Presence of fibroglandular structures}

In a more general and realistic scenario, breast tissue composition can be characterized as one of four types:

1) the breast is almost entirely fat;

2) there are scattered islands of fibroglandular tissue; 


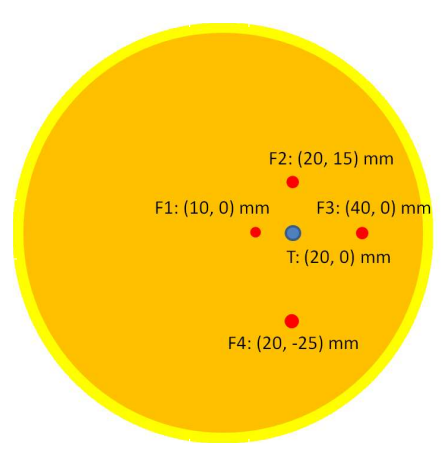

$\mathbf{a}$

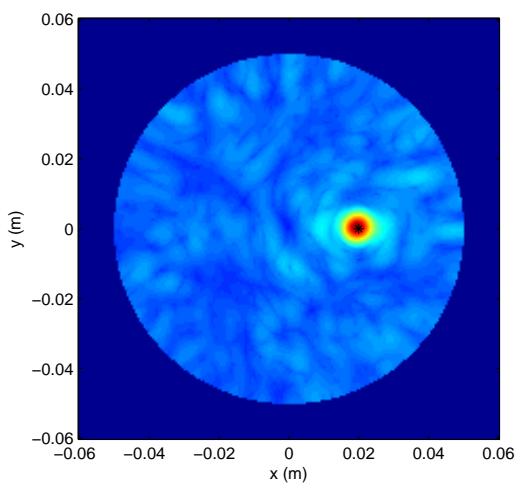

b
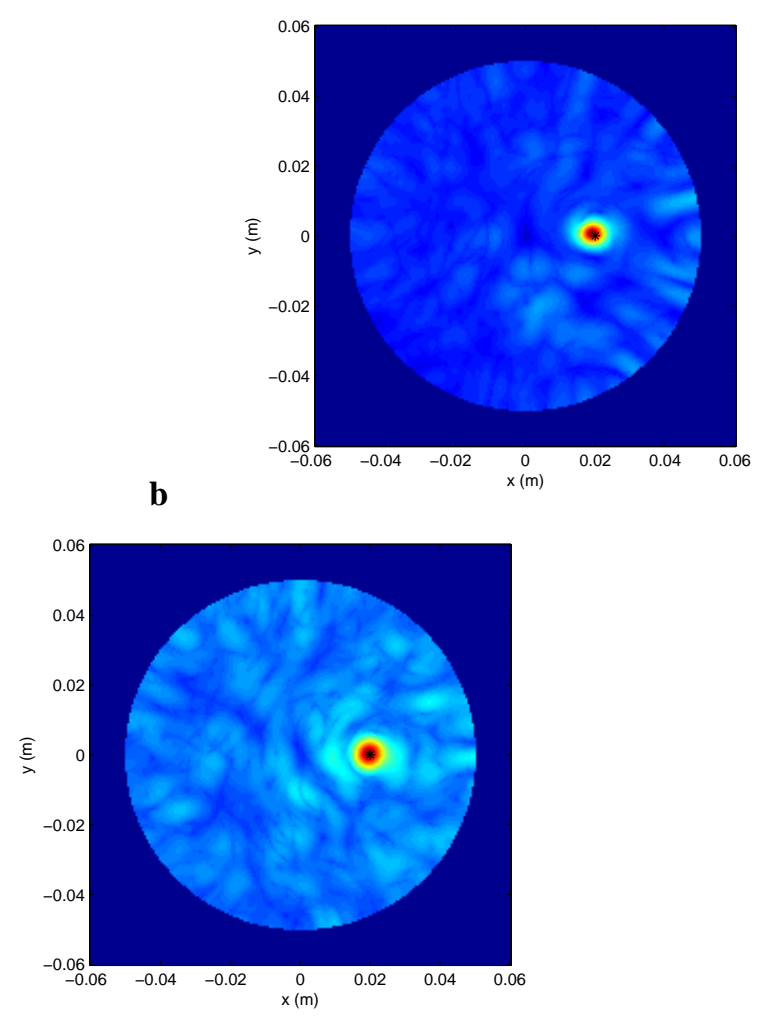

Fig. 5. a: Configuration layout; Tumor detection with FGS/breast contrast at b: $10 \%$; c: $30 \%$; d: $50 \%$.

3) the breast tissue is heterogeneously dense (which may lower the sensitivity of mammography);

4) the breast tissue is extremely dense (which will always lower the sensitivity of mammography).

At X-ray frequencies, breast cancers are radiodense (e.g., white on mammograms), radioluscent fat generally appears dark gray-to-black on mammograms and provides an excellent background in which to see small cancers. However, dense fibroglandular breast tissue can obscure small cancers [33]. For this reason, this type of healthy breast tissue must be considered in a more complete evaluation of a cancer detection system.

As type 1) scenarios have been considered in previous sections, the breast cancer detection is now performed in presence of fibroglandular structures. In particular, two scenarios were designed in order to take into account type 2) and 3) breast tissue distributions.

1) Islands of fibroglandular tissue: This scenario represents a stress condition for the algorithm as the FGSs present similar scattering behavior to the tumor and certain interference occurs among them. The tumor center estimation is reported in Fig. 5 (panels $b, c$ and $d$ ) and the corresponding metrics in Table II.

As can be seen, also for this case, the detection system is able to filter the scattered fields coming from the FGSs and properly localize the tumor.

TABLE II

METRICS FOR THE CASE ISLAND CUTTER.

\begin{tabular}{|c|c|c|c|c|}
\hline Metrics & FWHM $(\mathrm{mm})$ & SCR $(\mathrm{dB})$ & SMR $(\mathrm{dB})$ & SD $(\mathrm{mm})$ \\
\hline $10 \%$ & 4.8 & 10.3 & 15.3 & 0.98 \\
\hline $30 \%$ & 6 & 9.22 & 13.55 & 1 \\
\hline $50 \%$ & 6.4 & 8.14 & 12.34 & 1.1 \\
\hline
\end{tabular}

2) Large fibroglandular region: In this section, the main focus is still on testing the detection system in the presence of clutter which arises from fibrograndular structures. In this section an extended fibrograndular region that surrounds the tumor is considered. In particular, the tumor located at $(10,0) \mathrm{mm}$ is embedded within an ellipse whose axes and whose dielectric properties are varied. We start by considering a fibroglandular structure whose dielectric and conductivity properties 


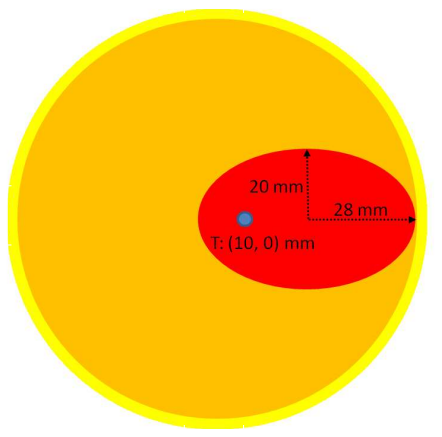

a

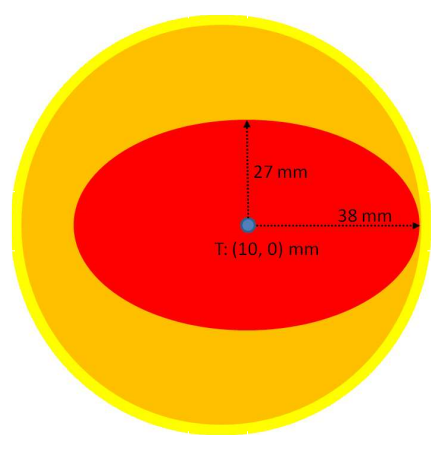

c

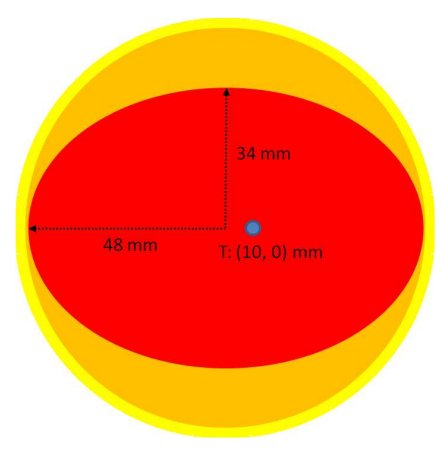

$\mathbf{e}$

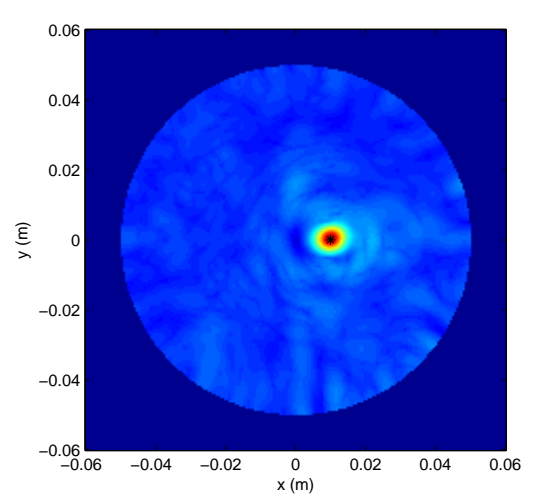

b

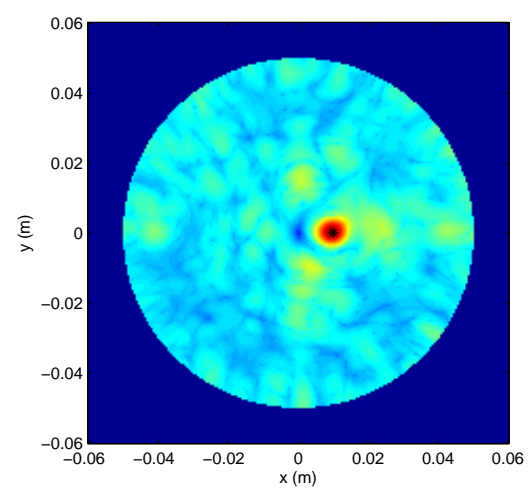

d

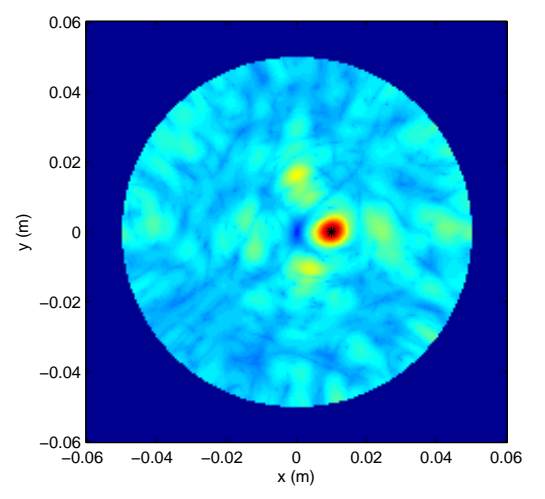

Fig. 6. Tumor detection with different FGS structure sizes.

are increased of $10 \%$ from those of normal breast tissue considered in the previous cases, whereas its size is varied as described in panels $a, c$ and $e$ in fig.6. The corresponding detection results are displayed in panels $b, d$ and $f$ of the same figure whereas the metrics are reported in Tab. III. As can be seen, the tumor is well detected in all cases. However, as expected, resolution and other metrics significantly reduce when the fibrograndular structure enlarges.

In order to assess the role of fibrograndular structure contrast, we now consider the same case as in panel $a$ of Fig. 6 . However, while the size of fibrograndular structure is kept fixed its contrast is further increased up to $50 \%$. Detection results and corresponding metrics are reported in Fig. 7 and Tab. IV. The system succeeds in detecting the small tumor in all investigated cases and the achievable performance are better than those shown for the previous case. At least for resolution, this can be expected. In fact, to obtain focussed reconstruction what is relevant is the mismatch between the assumed and the actual propagation path. This depends on the size as well as the contrast of the fibrograndular structure.

3) Island and large fibroglandular region: In this section we consider a fibrograndular structure that simultaneously includes extended and small formations of different size. These are randomly distributed within the breast tissue. Finally, different level of fibrograndular contrast are considered.

The layout of the configuration and the corresponding reconstructions are reported in Fig. 8, whereas Tab. V lists the relevant metrics.

As can be seen, it becomes more difficult to discern tumor against clutter artifacts (see values of SCR and SMR) than in 
TABLE III

METRICS FOR THE CASE OF LARGE FIBROGANDULAR REGION WITH VARYING SIZE.

\begin{tabular}{|c|c|c|c|c|}
\hline Metrics & FWHM $(\mathrm{mm})$ & SCR $(\mathrm{dB})$ & SMR $(\mathrm{dB})$ & SD $(\mathrm{mm})$ \\
\hline case a & 5.4 & 10.7 & 15.2 & 0.05 \\
\hline case b & 7.8 & 4.7 & 8.75 & 0.71 \\
\hline case c & 7.8 & 4.3 & 9.3 & 0.3 \\
\hline
\end{tabular}

TABLE IV

METRICS FOR THE CASE OF LARGE FIBROGANDULAR REGION WITH VARYING CONTRAST.

\begin{tabular}{|c|c|c|c|c|}
\hline Metrics & FWHM $(\mathrm{mm})$ & SCR $(\mathrm{dB})$ & SMR $(\mathrm{dB})$ & SD $(\mathrm{mm})$ \\
\hline $10 \%$ & 5.4 & 10.7 & 15.2 & 0.05 \\
\hline $30 \%$ & 7.2 & 4.2 & 8.3 & 1.3 \\
\hline $50 \%$ & 7.8 & 3.69 & 7.810 .2 & 0.6 \\
\hline
\end{tabular}

previous cases. However, by comparing reconstructions of Fig. 8 and the ones corresponding to the same scenario but obtained by background subtraction (not reported here) we found that this is mainly due to the adopted clutter rejection method.

\section{CONClusions}

A novel RF breast cancer detection system has been introduced in this paper. The system components consisting of a simplified phantom, a MUSIC-inspired detection algorithm and a ParEGO Vivaldi antenna have been described in detail. A simplified 2-D phantom was used to assess the performance, while the system was implemented with a realistic antenna and tested in different critical scenarios. Together with visual reconstruction of the detection algorithm results, its performance was measured by suitable metrics such as Full-Width-Half-Maximum (FWHM), Signal-to-Clutter Ratio (SCR), Signal-to-Mean Ratio (SMR) and Spatial displacement (SD). Detection was successful in the presence of a single 5-mm tumor when positioned at different distances from the skin layer. But also when 3 tumoral in-homogeneities are introduced the detecting system is proven to be successful. Finally, the system was tested in the presence of fibro-glandular structures (FGS) that strongly reduce the dielectric contrast between benign and malignant tissues. For both scattered and large FGS, the tumor was correctly detected with a high degree of precision. In conclusion, the proposed RF detection system here presented offers encouraging performance for early-stage breast cancer diagnostics. Its MUSIC-like algorithm proves to be suitable for a non-ideal antenna and performs correctly in critical scenarios. In particular, this algorithm does not require any antenna calibration or phase response estimation. The employment of efficient and complex antenna geometries is allowed without difficult algorithm redefinitions. In fact, multifrequency data are not used for de-correlation purposes [29] or to construct a wideband estimator [34]. In those cases, data might be coherent and thus information about the antenna would be necessary. Alternatively, we separately performed different reconstructions at each frequency. This eliminates the necessity of a priori antenna phase response characterization. Moreover,

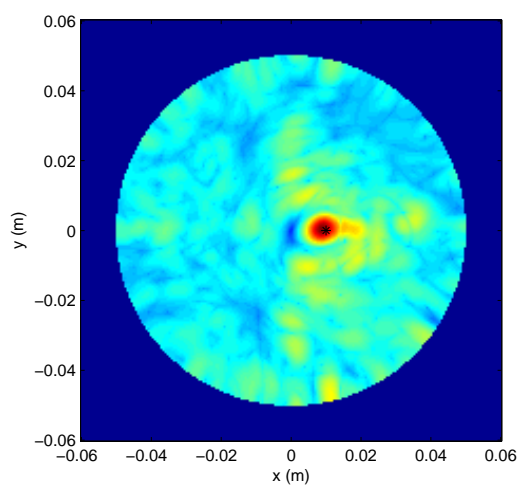

a

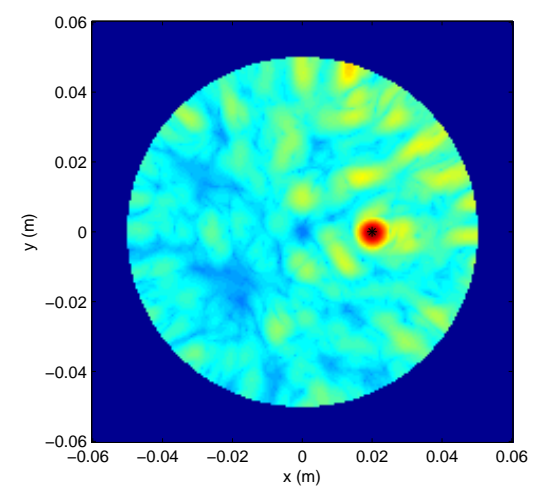

Fig. 7. Tumor detection with FGS/breast contrast at a: $30 \%$; b: $50 \%$; 


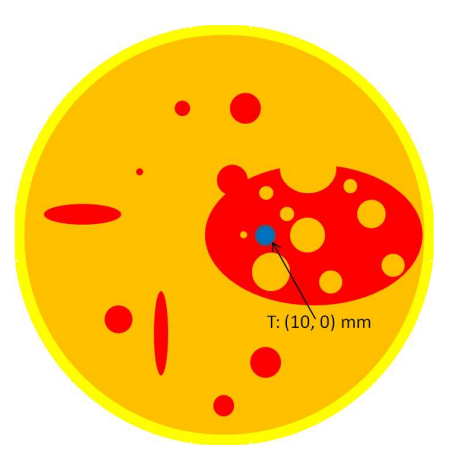

a

c

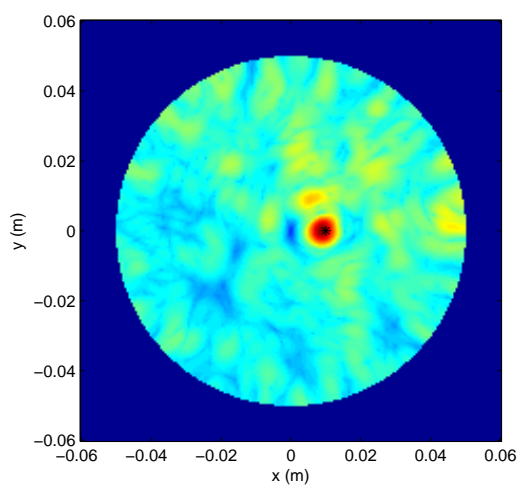

b
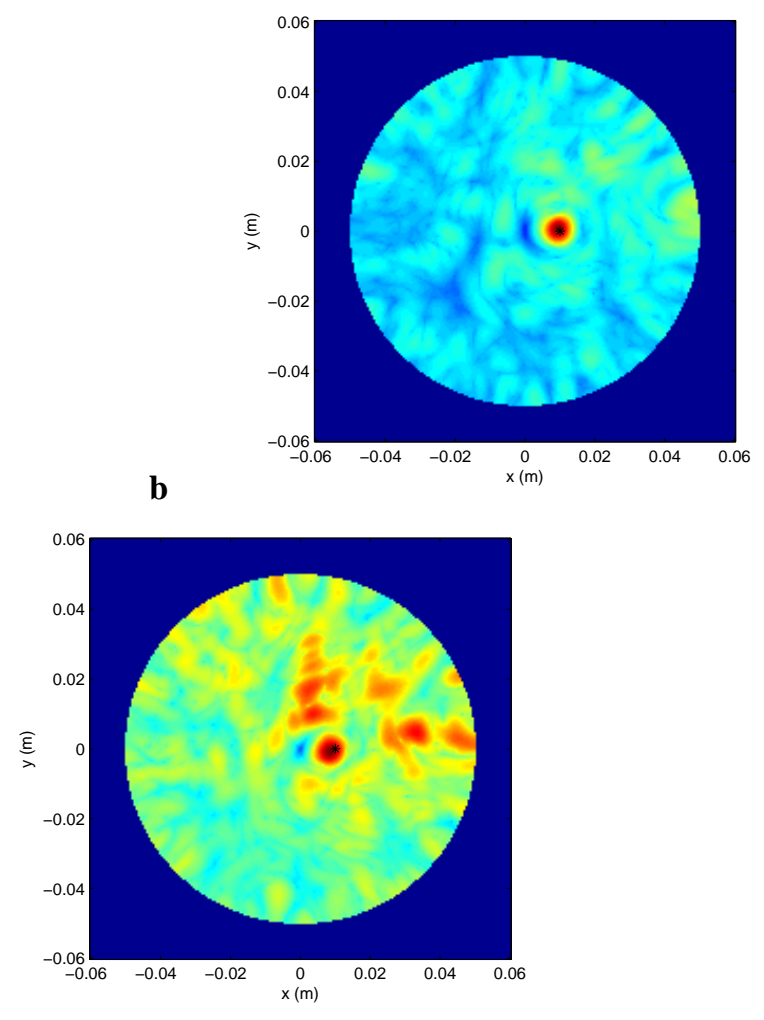

Fig. 8. a: Configuration layout; Tumor detection with FGS/breast contrast at b: 10\%; c: $30 \%$; d: $50 \%$.

TABLE V

METRICS FOR THE CASE OF LARGE ISLAND PLUS FIBROGANDULAR REGION WITH VARYING CONTRAST.

\begin{tabular}{|c|c|c|c|c|}
\hline Metrics & FWHM $(\mathrm{mm})$ & SCR $(\mathrm{dB})$ & SMR $(\mathrm{dB})$ & SD $(\mathrm{mm})$ \\
\hline $10 \%$ & 5.8 & 5.26 & 8.9 & 0.8 \\
\hline $30 \%$ & 5.8 & 3.97 & 7.71 & 2.2 \\
\hline $50 \%$ & 5.84 & 1.91 & 5.71 & 2.32 \\
\hline
\end{tabular}

the broadband antenna requirement is more relaxed compared to other UWB approaches, as the system operates on a discrete set of frequencies rather than on continuous spectrum.

\section{ACKNOWLEDGMENT}

This work was supported by the Dublin Institute of Technology and the COST Action IC0603 ASSIST. The authors would like to acknowledge Dr. Matthias John for his ParEGO optimization tool.

\section{REFERENCES}

[1] P. T. Huynh, A. M. Jorolimek, and S. Dave, The False-negative mammogram, Radiograph, Vol. 18, no. 5, 1988 , pp. $1137-1154$.

[2] A. J. Surowiec, S. S. Stuchly, J. R. Barr, and A. Swarup, Dielectric properties of breast carcinoma and the surrounding tissues, IEEE Trans. Biomed. Eng., Vol. 35, Apr. 1988, pp. 257-263.

[3] D. Colton, and R. Kress Inverse Acoustic and Electromangetic Scattering Theory, Springer-Verlag, 1992, Berlin.

[4] M. Donelli, I. Craddock, D. Gibbins, and M. Sarafianou, A Three-Dimensional Time Domain Microwave Imaging Method for Breast Cancer Detection Based on an Evolutionary Algorithm, Progress In Electromagnetics Research M, Vol. 18, 2011, 179-195.

[5] T. Isernia, V. Pascazio, and R. Pierri, On the local minima in a tomographic imaging technique, IEEE Trans. Geosc. Rem. Sens., Vol. 39, 2011, pp. 1596-1607.

[6] D. Byrne, M. O’Halloran, M. Glavin, and E. Jones, Data Independent Radar Beamforming Algorithms for Breast Cancer Detection, Progress In Electromagnetics Research, Vol. 107, 2010, pp. 331-348.

[7] P. Kosmas, and C. M. Rappaport, A Matched-Filter FDTD-Based Time Reversal Approach for Microwave Breast Cancer Detection, IEEE Trans. Antennas Propag., Vol. 54, no. 4, Aug. 2006, pp. 1257-1264.

[8] R. Solimene, A. Brancaccio, J. Romano, and R. Pierri, Localizing Thin Metallic Cylinders by a 2.5-D Linear Distributional Approach: Experimental Results, IEEE Trans. Antennas Propag., Vol. 56, no. 8, Aug. 2008, pp. 2630-2637. 
[9] R. C. Conceicao, M. O’Halloran, E. Jones, and M. Glavin, Investigation of Classifiers for Early-Stage Breast Cancer Based on Radar Target Signatures, Progress In Electromagnetics Research, Vol. 105, 2010, pp. 295-311.

[10] M. Lazebnik, L. McCartney, D. Popovic, C. B. Watkins, M. J. Lindstrom, J. Harter, S. Sewall, A. Magliocco, J. H. Booske, M. Okoniewski, and S. C. Hagness, A large-scale study of the ultrawideband microwave dielectric properties of normal breast tissue obtained from reduction surgeries, Phys. Med. Biol., Vol. 52, 2007, pp. 2637-2656.

[11] M. Lazebnik, D. Popovic, L. McCartney, C. B. Watkins, M. J. Lindstrom, J. Harter, S. Sewall, T. Ogilvie, A. Magliocco, T. M. Breslin, W. Temple, D. Mew, J. H. Booske, M. Okoniewski, and S. C. Hagness, A large-scale study of the ultrawideband microwave dielectric properties of normal, benign and malignant breast tissues obtained from reduction surgeries, Phys. Med. Biol., Vol. 52, 2007, pp. 6093-6115.

[12] P. M. Meaney, K. D. Paulsen, and J. T. Chang, Near-Field Microwave Imaging of Biologically-Based Materials Using a Monopole Transceiver System, IEEE Trans. Microwave Theory Tech., Vol. 46, no. 1, 1998, pp. 31-45.

[13] G. Ruvio, R. Solimene, and M. J. Ammann, Evaluation of Antenna Types for RF Breast Cancer Imaging using 2-Layer Planar Tissue Model, Proc. of 40th European Microwave Conference, Sept 2010, pp. 212-215.

[14] S. M. Salvador, and G. Vecchi, Experimental tests of microwave breast cancer detection on phantoms, IEEE Trans. Antennas Propag., Vol. 57, no. 6, June 2009, pp. 1705-1712.

[15] X. Li, S. C. Hagness, M. K. Choi, and D. W. Van der Weide, Numerical and Experimental Investigation of an Ultrawideband Ridged Pyramidal Horn Antenna With Curved Launching Plane for Pulse Radiation, IEEE Antennas and Wireless Propag. Letters, Vol. 2, 2003, pp. $259-262$.

[16] D. Gibbins, M. Klemm, I. J. Craddock, J. A. Leendertz, A. Preece, and R. Benjamin, A Comparison of a Wide-Slot and a Stacked Patch Antenna for the Purpose of Breast Cancer Detection, IEEE Trans. Antennas Propag., Vol. 58, no. 2, March 2010, pp. 665-674.

[17] M. Klemm, J. A. Leendertz, D. Gibbins, I. J. Craddock, A. Preece, and R. Benjamin, Microwave Radar-Based Differential Breast Cancer Imaging: Imaging in Homogeneous Breast Phantoms and Low Contrast Scenarios, IEEE Trans. Antennas Propag., Vol. 57, no. 7, April 2010, pp. 2337-2344.

[18] B. J. Mohammed, A. M. Abbosh, and P. Sharpe, Planar Array of Corrugated Tapered Slot Antennas for Ultrawideband Biomedical Microwave Imaging System, International Journal of RF and Microwave Computer-Aided Engineering, pp. 1-8, 2012.

[19] M. John, M. J. Ammann, and P. McEvoy, UWB Vivaldi antenna based on a spline geometry with frequency band-notch, IEEE AP-S Intern. Symp. Antennas and Propag. 2008, Session 412, Paper 7.

[20] M. John, and M. J. Ammann, Antenna Optimisation with a Computationally Efficient Multi-objective Evolutionary Algorithm, IEEE Trans. Antennas Propag., Vol. 57, no. 1, January 2009, pp. 260-263.

[21] R. O. Schmidt, Multiple emitter location and signal parameter estimation, IEEE Trans. Antennas Propag., Vol. 34, 1986, pp. $279-280$.

[22] G. Ruvio, R. Solimene, A. D’Alterio, M. J. Ammann, and R. Pierri, Radio-Frequency Breast Cancer Imaging Results for a Simplified Cylindrical Phantom, Proc. of 10th International Congress of the European Bioelectromagnetic Association (EBEA), Rome, Italy, Feb. 2011.

[23] J. Bourqui, M. Okoniewski, and E.C. Fear, Balanced Antipodal Vivaldi Antenna with Dielectric Director for Near-Field Microwave Imaging, IEEE Trans. on Antennas and Propag., Vol. 58, No. 7, July 2010, pp. 2318-2326.

[24] R. C. Coincecao, M. O'Halloran, M. Glavin, and E. Jones, Comparison of planar and circular antenna configurations for breast cancer detection using microwave imaging, Progress In Electromagnetics Research, Vol. 99, 2009, pp. 1-20.

[25] Y. Chen, E. Gunawan, K. Soon Low, S-C. Wang, C. Boon Soh, and T. Choudary Putti, Time-Reversal Ultrawideband Breast Imaging: Pulse Design Criteria Considering Multiple Tumors With Unknown Tissue Properties, IEEE Trans. Antennas Propag., Vol. 56, no. 9, September 2008, pp. 3073-3077.

[26] E. J. Bond, X. Li, S. C. Hagness, and B. D. Van Veen, Microwave imaging via space-time beamforming for early detection of breast cancer, IEEE Trans. Antennas Propag., Vol. 51, 2003, pp. 1690-1705.

[27] J. Knowles, ParEGO: A hybrid algorithm with on-line landscape approximation for expensive multiobjective optimization problems, IEEE Trans. Evol. Comput., Vol. 10, no. 1, February 2005, pp. 50-66.

[28] T. B. Hansen and A. D. Yaghjian, Low-frequency scattering from two dimensional perfect conductors, IEEE Trans. Antennas Propag., Vol. 40, 1992, pp. 1389-1402.

[29] J. W. Odendaal, E. Barnard, and C. W. I. Pistorious, Two-Dimensional Super-Resolution Radar Imaging Using the MUSIC Algorithm, IEEE Trans. Antennas Propagat., Vol. 42, 1994, pp. 1386-1391.

[30] F. K. Gruber, E. A. Marengo, and A. J. Devaney, Time-reversal imaging with multiple signal classification considering multiple scattering between the targets, J. Acoust. Soc. Am., Vol. 115, 2004, pp. 3042-3047.

[31] K. Agarwal, L. Pan, Y. K. Leong, M. Han, O. Y. Chan, X. Chen, and S. P. Yeo, Practical Application of Multiple Signal Classification, International Journal of RF and Microwave Computer-Aided Engineering, Vol. 22, May 2012, pp. 359-369.

[32] [Online], www.cst.com

[33] D. W. Kufe, R. E. Pollock, R. R. Weichselbaum, R. C. Bast, T. S. Gansler, J. F. Holland, and E. Frei, Cancer Medicine, 6th Edition, BC Decker Inc., Hamilton $(\mathrm{ON}), 2003$.

[34] Y. S. Yoon, L. M. Kaplan, and J. H. McClellan, TOPS: New DOA estimator for wideband signals, IEEE Trans. Sign. Process., Vol. 54, no. 6, 2006, pp. 1977-1989. 